Supporting Information

\title{
Carbon Nanotubes Contain Residual Metal Catalyst Nanoparticles Even after Washing with Nitric Acid at Elevated Temperature Because These Metal Nanoparticles Are Sheathed by Several Graphene Sheets
}

\author{
Martin Pumera* \\ Dr. M. Pumera, ICYS, National Institute for Materials Science, 1-1 Namiki, Tsukuba, \\ Ibaraki, Japan. Phone: +81-29-851-3354-8816; fax: +81-29-860-4706, email: \\ PUMERA.Martin@nims.go.jp
}

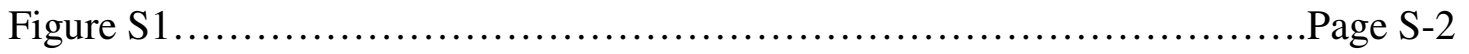





Figure S1. TEM images of as-received multi wall and single wall carbon nanotube materials before 'washing' with concentrated nitric acid at $80^{\circ} \mathrm{C}$. 